\title{
An Algorithm for Video-Polysomnography Method to Create Shortened Motion Video from Whole Night Sleep Video
}

\author{
K M Talha Nahiyan, Md. Zahidul Islam, Md. Adnan Kiber \\ Department of Applied Physics, Electronics \& Communication Engineering, University of Dhaka \\ Dhaka, Bangladesh
}

\begin{abstract}
A sleep disorder is a medical disorder of sleep patterns of a person. Polysomnography is usually used to determine sleep disorder but it is a partly invasive and interfering method. Thus anyone having this test may face difficulty in having sleep during the procedure and may result in inappropriate diagnosis. In case of abnormal motion related sleep disorder, a video-polysomnography method can be used where the whole night sleep is recorded and analyzed later. But a whole night sleep video is 6-8 hours long and thus very difficult to examine such a lengthy video. In this paper it is proposed that if we create a shortened motion video out of the whole night sleep video, containing only the movement related portions, then a sleep analyst can diagnose for sleep disorder by analyzing that video in a short time and order further extensive test. For primary detection of sleep disorder it can be a low cost, simply implemented and noninvasive method of video-polysomnography.
\end{abstract}

\section{General Terms}

Image processing, Video processing, Video-polysomnography

\section{Keywords}

Video-polysomnography, Shortened motion video

\section{INTRODUCTION}

Polysomnography is a test to diagnose sleep disorder of a person. A typical polysomnography usually requires a minimum of 12 channels requiring a minimum of 22 wire attachments to the patient. It is interfering and partly invasive due to wiring and electrodes required for measuring EOG, EEG, ECG and EMG [1] [2]. Thus a person already having some kind of sleep disorder may even face further difficulty to sleep. Video-polysomnography has been used along with conventional polysomnography where the whole night sleep is recorded and analyzed along with other parameters. When the particular interest is to detect any abnormal movement related sleep disorder then only the sleep video can be a useful aid, eliminating the need for conventional polysomnography initially. This can allow a noninvasive and non-interfering method to analyze sleep disorder. But the usual sleep video of 6-8 hour duration is difficult to watch for a sleep analyst due to its long length. Moreover most of the duration of the video would be of normal sleep with no significant movement. If the video can be processed to create a shortened video, consisting only of the motion parts then it would be easier to analyze. So the goal is to develop an algorithm using motion detection technique to create a shortened motion video out of the whole night sleep video.

\section{VIDEO RECORDING CONDITIONS}

The recorded video to be processing eligible three important conditions are to be met. Firstly, the light intensity in the room should be flicker free and kept at a constant intensity level. Secondly, the background environment should be stationary not causing any unwanted movements. Finally the camera should be fixed at a suitable position.

\section{ALGORITHM DEVELOPMENT FOR PROCESSING SLEEP VIDEO}

The MATLAB ${ }^{\circledR}$ and it image processing toolbox is used to create the code which can process the sleep video. In the following sections motion detection technique, the algorithm and flowchart is discussed.

\subsection{Motion Detection Technique}

There are various techniques to detect motion in a video. The difference image technique is one of them. First of all the video frames will be considered as images and then by performing absolute image difference on the two images it can be decided that whether there is any motion. For this the "imabsdiff" function in MATLAB ${ }^{\circledR}$ can be used. If $X$ and $Y$ are two images of same class and size then $\mathrm{Z}=$ imabsdiff $(\mathrm{X}, \mathrm{Y})$ subtracts each element in array $\mathrm{Y}$ from the corresponding element in array $X$ and returns the absolute difference in the corresponding element of the output array $\mathrm{Z}$. Z has the same class and size as $X$ and $Y$ [3][4]. If two subsequent frames in a video are same then it would result in a matrix $Z$ with all elements zero. But if there is motion between the frames then the matrix $\mathrm{Z}$ would contain intensity values. This is the basis of our motion detection to extract frames of the video.

\subsection{Algorithm and Flowchart}

To create the desired shortened motion video, first the video file have to be read and a file is also created to save the output. Then subsequent frames are read and compared to determine whether motion is present or not. Frames which contain motion are then added to create the output video.

The function mmreader was used to read a video file. $o b j=$ mmreader(filename) constructs obj to read video data from the file named filename. To read frames the function read was used in collaboration with mmreader. video $=\operatorname{read}(o b j$, index $)$ reads only the specified frames. index can be a single number or a two-element array representing an index range of the video stream. A video file was created by adding only the 


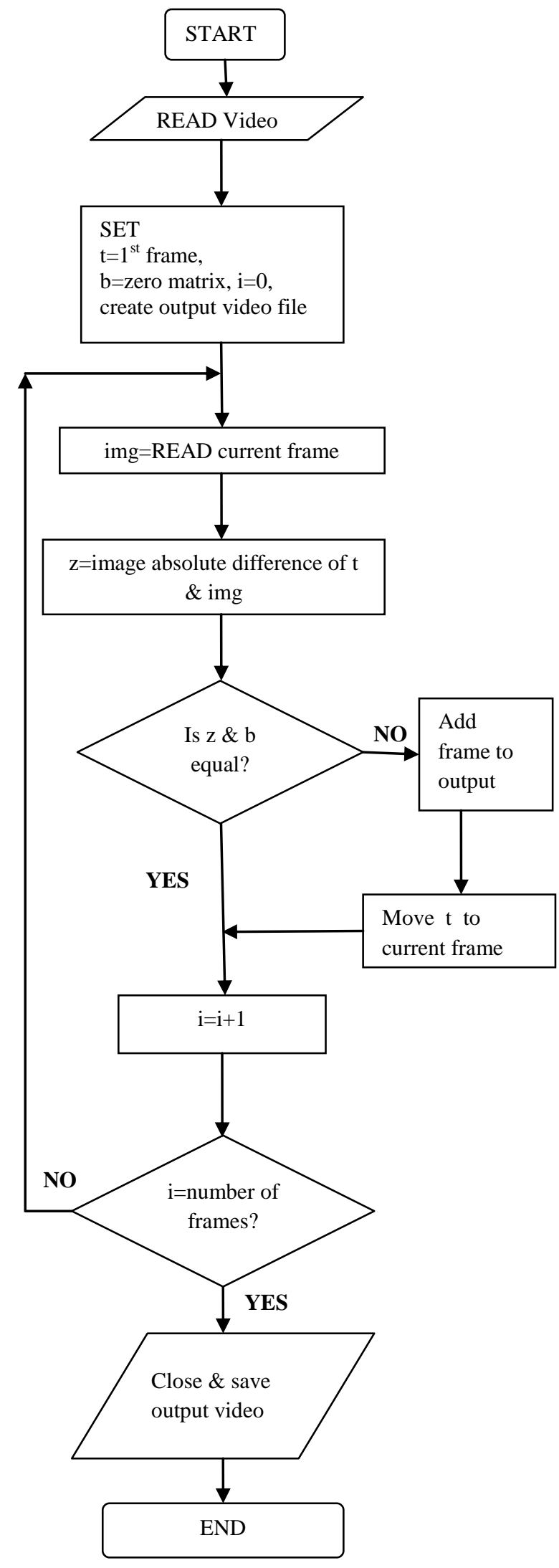

Figure 1. Flowchart to create shortened motion video motion frames. The function avifile creates a video file in AVI format and addframe function allows adding frames selectively. aviobj = avifile(filename) creates an avifile object, giving it the name specified in filename, using default values for all avifile object properties. aviobj = addframe (aviobj,frame) appends the data in frame to the AVI file identified by aviobj, which was created by a previous call to avifile. frame can be either an indexed image (m-by-n) or a truecolor image (m-by-n-by-3) of double or uint8 precision [5].

The subsequent frames are compared to find out motion. Initially the first frame is set as the template with which the next frame is compared. Absolute image difference of two frames results in a zero matrix if there is no motion. The result is compared with a zero matrix to determine motion. If motion is present then the template frame is moved to current frame and the current frame is also added to output video. The loop continues until all frames are compared. At the end of this process an output video is created containing the motion frames. The algorithm is as follows:

START

STEP 1: Read video file (using mmreader)

STEP 2: Set, $\mathrm{t}=1^{\text {st }}$ frame,

$b=$ zero matrix (of same size \& class of frames), create a video file (using avifile)

STEP 3: FOR $i=1$ :Number Of Frames, img $=$ Read current frame,

Do image absolute difference of $\mathrm{t} \&$ img (using imabsdiff),

Compare $\mathrm{z} \& \mathrm{~b}$, Is $\mathrm{z} \& \mathrm{~b}$ equal?

IF yes then read next frame

IF no then add frame to output file (using addframe ) and move $t$ to next frame

STEP 4: save and close output video file.

END

The flowchart for this algorithm is shown in figure 1.

\section{COMPARISON OF PROPOSED METHOD WITH EXISTING TECHNIQUES}

This section provides a comparative discussion of typical polysomnography and video polysomnography. Also it mentions the advantages of the proposed videopolysomnography method with compared to conventional video-polysomnography.

\subsection{Typical Polysomnography \& Video- polysomnography}

As discussed earlier a typical polysomnography requires a minimum 22 wire attaching electrodes to the patient which is used for monitoring EEG, EOG, ECG and EMG. Thus it is a partly invasive and interfering method. Whereas videopolysomnography requires no devices or electrodes attached to the body of the patient. So it provides a noninvasive and non-interfering method.

\subsection{Proposed Method \& Conventional Video-Polysomnography}

Though video-polysomnography offers a noninvasive and non-interfering way of diagnosing sleep disorder, it has a major disadvantage. The whole night sleep video is $6-8$ hours long and makes it difficult for a sleep analyst to examine such 
a long video. The proposed method of videopolysomnography can overcome this problem. The algorithm used in this method creates a short video containing only the motion parts of the original video by applying motion detection algorithm. Only the motion parts of the video are of interest and thus creating a short motion video introduces a more efficient method than conventional polysomnography.

So it can be said that the proposed method can improve the existing video-polysomnography method and replace polysomnography as an initial diagnosis of sleep disorder. Still polysomnography remains the gold standard test to diagnose sleep disorder but the proposed method can be used when noninvasive and non-interfering properties are priority such as in case of children [6] [7]. It also offers a cost effective and simple to implement method that can be easily installed.

\section{RESULT \& ANALYSIS}

It was unable to process a whole sleep video due the size of such a video and huge processing time and capability needed. A mock sleep video of 4 minutes 45 seconds was recorded. After processing the video a shortened motion video of 37 seconds was produced. The information of these videos are given in table 1.Though a whole sleep video was not processed but the applied algorithm works. This method has great potential, simple to implement and future work can be done to improve it.

Table 1. Information of original and processed video

\begin{tabular}{|l|l|l|}
\hline & Original Video & Processed Video \\
\hline Filename & 'sample.avi' & 'motvideo.avi' \\
\hline Duration & 4 min 45 seconds & 37 seconds \\
\hline Resolution & $640 \times 480$ & 640 x 480 \\
\hline Image type & Truecolor & Truecolor \\
\hline
\end{tabular}

\section{CONCLUSION}

This method has some limitations and future work can be done on reducing the processing time and making the method more accurate by introducing filtering and motion compensation. By adding motion compensation technique and filtering the need for video recording conditions can be eliminated. Thus it would be able to record video for processing in any circumstance regardless of room, light and camera conditions. Moreover if action recognition can be added to this then it would be possible to determine the nature of motion and indicating towards specific sleep disorder might become a possibility. Further modifications can be done if tested clinically and assessing the outcomes in collaboration with a sleep analyst.

\section{REFERENCES}

[1] http://en.wikipedia.org/wiki/Polysomnography

[2] K. E. Bloch, "Polysomnography: A systematic review," Technol. Health Care, vol. 5, no. 4, pp. 285-305, Oct. 1997

[3] Image Processing Toolbox ${ }^{\mathrm{TM}}$ User's Guide, The Mathworks, Inc.

[4] Rafael C. Gonzalez, Richard E. Woods and Steven L. Eddins (2004), Digital Image Processing using MATLAB, Pearson Education.

[5] Data Import and Export User's Guide, The Mathworks, Inc.

[6] Kayyali H, Weimer S, Frederick C, et al. "Remotely attended home monitoring of sleep disorders." Telemedicine and E-health. May 2008. Vol 14(4):371-4.

[7] Banno K, Kryger MH. "Use of polysomnography with synchronized digital recording to diagnose pediatric sleep breathing disorders." JAMC 2005;173(1)28-30. 